\title{
Valoraciones empírico-teóricas sobre los métodos de evaluación de la transferencia de carga eléctrica en la soldadura con electrodo revestido
}

\author{
(Theoretical and empirical assessment of evaluation methods of the electric charge transference in welding with covered \\ electrodes)
}

\author{
Alejandro García Rodríguez ${ }^{1}$, Jorge V Miguel Oria ${ }^{1}$, Alexis Delgado Gómez \\ ${ }^{1}$ Universidad Central "Marta Abreu" de las Villas, Centro de Investigaciones de Soldadura (CIS) /Ministerio de Educación Superior \\ (MES), Santa Clara, Villa Clara, Cuba,alejo2@uclv.edu.cu
}

\begin{abstract}
Resumen
El presente trabajo se basa en la valoración de resultados obtenidos experimentalmente, mediante la aplicación de diferentes métodos de evaluación, reportados en la literatura especializada, de la estabilidad en el proceso de transferencia de carga eléctrica a través del arco. Un diseño factorial simple, cuya variable independiente es la corriente de soldadura, fue aplicada al proceso con electrodos AWS A5.1 E6013. Los depósitos de soldadura fueran realizados en posición plana con el empleo de un dispositivo de alimentación por gravedad. Fue obteniendo mediante procesamiento digital de señales y técnicas estadísticas, criterios sobre la estabilidad del proceso de transferencia de carga eléctrica, refrendados mediante la aplicación de técnicas de inspección visual y estudio de la morfología. De los resultados obtenidos, se concluye que entre los parámetros considerados para valorar el proceso de transferencia de carga eléctrica la mediana de la conductividad de los picos de reencendido y la cantidad de picos de reencendido se presentan como los índices más representativos, tanto desde el punto estadístico como fenomenológico. Esto se corrobora en el hecho de que el estudio morfológico metalográfico y el análisis de estabilidad del proceso de transferencia de carga eléctrica coincide en que el régimen de $160 \mathrm{~A}$ (para electrodos de $4 \mathrm{~mm}$ de diámetro) es el más estable, comprobándose la validez del método empleado.
\end{abstract}

Palabras-Clave: Soldadura. Procesamiento digital. Estadístico.

Abstract: The present work is based on an assessment of experimentally obtained results from the application of different methods of evaluation, reported in the specialized literature, of the electrical charge transference stability in the arc. A simple factorial design, taking the welding current as independent variable, was applied to the process with an AWS A5.1 E6013 electrode. The welds were carried out in the flat position by a gravity device. It was possible to obtain criteria to classify the electrical charge transference stability of the process, by digital signal processing and statistical technique. The outcome was based on visual inspection and morphologic studies. It was concluded that amongst the considered parameters the average value of the arc conductivity during re ignition peaks and the quantity of re ignition peaks are the more representative indexes, both from statistics and from the phenomenological points of view. This finding is validated by the fact that the bead morphology and the stability analysis of the electrical charge transference process coincides with the fact that the $160 \mathrm{~A}$ (for 4-mm-diameter electrodes) is the most stable regime.

Key-Words: Welding. Digital Processing. Statistic

\section{Introducción}

La estabilidad general en procesos de soldadura por arco eléctrico constituye un indicador importante para el control de la calidad del proceso, cuyo monitoreo en tiempo real puede realizarse mediante el empleo de técnicas de digitalización, registro, procesamiento digital y estadístico de las señales de voltaje y corriente del arco de soldadura; especialmente en lo

Recebido em 29/05/2014, texto final em 26/09/2014.

DOI: http://dx.doi.org/10.1590/0104-9224/SI1903.10 referido al índice de transferencia de carga eléctrica [1].

La compleja fenomenología del arco eléctrico de soldadura se inicia cuando la atmósfera gaseosa protectora generada y excitada por el intenso gradiente energético producido principalmente por efecto Joule, en el momento final del cortocircuito del electrodo con la pieza a soldar, alcanza la temperatura suficiente para que se sostenga el proceso de ionización de las especies químicas de más bajo potencial de ionización, contenidas en el espacio inter electródico. La reacción en cadena debe propiciar un incremento progresivo de la temperatura y por tanto del grado de ionización de las sustancias, estableciendo un plasma térmico que alcanza temperaturas superiores a los $6000 \mathrm{~K}$ y que puede alcanzar cerca de los $12000 \mathrm{~K}$ en determinados procesos y aplicaciones; constituyendo la fuente de suministro de energía para la fusión de los metales a soldar $[2,3]$. 
La facilidad de establecimiento y restablecimiento del plasma se manifiesta en la capacidad que el proceso tecnológico tiene para recuperarse de los efectos de la ocurrencia de eventos desestabilizadores producidos aleatoriamente por diversos factores, entre los que se han reportado en la literatura especialmente: procesos de transferencia metálica a través del arco, características dinámicas de la fuente de soldadura, magnitudes de las variables esenciales del proceso y naturaleza de los materiales a soldar, entre otras [4].

La evaluación de la estabilidad de la transferencia de carga en la soldadura por arco eléctrico ha sido tratada en la literatura especializada [1, 5, 6, 7], planteándose métodos más o menos utilizados en trabajos científicos sobre el tema: Método A, propuesto por Farías [5]; Método B, propuesto por Ponomarev [7] y Método C, propuesto por Alejandro [1]), que es necesario comparar, comprobando su efectividad como criterio de estabilidad del proceso.

Oscilogramas obtenidos (Figura 1) en la soldadura con electrodo revestido y dispositivo de alimentación por gravedad en cada uno de los experimentos realizados, se tendrán en cuenta durante la evaluación del proceso de transferencia de carga a través del arco.

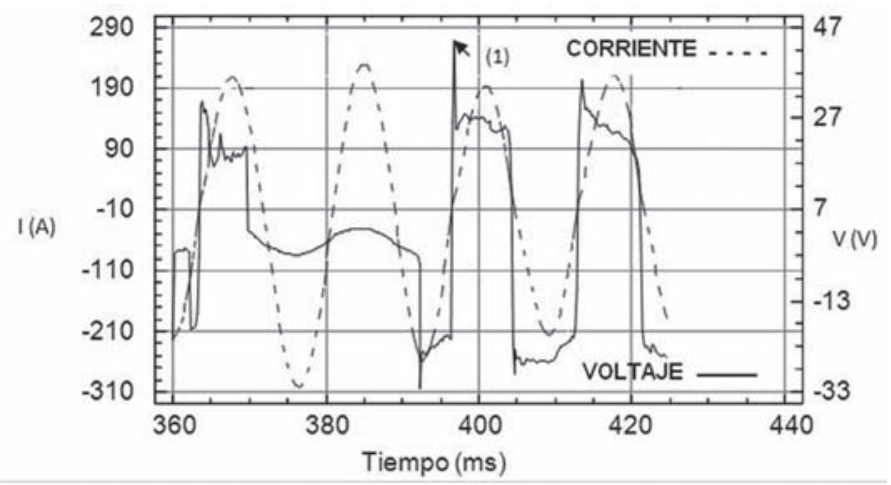

Figura 1. Oscilogramas de voltaje (en línea continua) y corriente (línea discontinua) sincronizados en tiempo de la soldadura con electrodos revestidos (Corriente Alterna (CA, 60 $\mathrm{Hz})$ )

\section{Método A (Farias)}

Souza [8] validó un sistema computarizado basado en una metodología propuesta por Farias [5] para valorar el desempeño operativo de electrodos revestidos según el cuál un arco estable debería cumplir dos órdenes de requisitos: primero, transferencia de metal fácil y uniforme; segundo, transferencia de carga eléctrica fácil y uniforme.

Para el caso de la transferencia de carga en la soldadura con corriente alterna, define el Índice $B^{+}$(Ecuación 1) que representa la conductividad eléctrica promedio en el espacio inter electródico durante el período de pre-arco positivo o pico de reencendido por su unidad de tiempo de duración, en la soldadura CA ((1) Figura 1).

$$
B^{+}=\frac{I^{+}}{U^{+} t^{+}} \cdot 1000 \quad\left(\Omega^{-1} s^{-1}\right)
$$

donde: $U^{+}$- Tensión promedio de reapertura del arco (V); $I^{+}-$
Corriente promedio de reapertura del arco (A); $t^{+}$- Tiempo de reapertura (s)

Según Farías el coeficiente de regularidad del transporte de carga eléctrica se calcula como el inverso del coeficiente de variabilidad de los parámetros en estudio para corriente alterna (Ecuación 2):

$$
R B^{+}=\frac{\overline{B^{+}}}{\sigma_{B^{+}}}
$$

donde: $B^{+}$, es el valor medio del índice $B^{+}$, y $\sigma_{B+}$, es la desviación estándar de este parámetro, definida por la Ecuación 3 donde n es la cantidad de picos de reencendido analizados.

$\sigma_{B+}=\sqrt{\frac{\sum_{i=1}^{n}\left(B_{i}-\overline{B^{+}}\right)^{2}}{(n-1)}}$

Como se aprecia (Ecuación 2) el coeficiente de regularidad es el inverso del coeficiente de variabilidad que se encuentra en función de la desviación típica de $\mathrm{B}^{+}$respecto al valor promedio del conjunto de valores de $\mathrm{B}^{+}$. La comparación efectiva del comportamiento eléctrico durante el reencendido de electrodos con valores promedios de $\mathrm{B}^{+}$, con medias diferentes, se encuentra inhabilitada matemáticamente si se emplean estas expresiones y restringida a la distribución normal de dichos valores [1].

Para Ponomarev [7] la estabilidad del arco depende de la magnitud del voltaje aplicado al espacio en que se establece el arco al comienzo de cada semiciclo, llamado voltaje de recuperación o reencendido, el cual debe superar el voltaje de necesario para el reencendido del arco.

\section{Método B (Ponomarev)}

Una condición fidedigna del reencendido del arco en corriente alterna (y, por tanto, de estabilidad del arco) puede ser la siguiente: el reencendido del arco CA es seguro si se suministra un voltaje de circuito abierto $U_{o . c}^{a}$, cuya amplitud sea igual o mayor que el voltaje máximo de reencendido durante el semiciclo positivo ( $U_{r . i+}$ es el voltaje el pico marcado con el número ((1) en la Figura $\stackrel{r}{\perp})$. Esta condición puede expresarse según la Ecuación 4:

$U_{o . c}^{a} \geq U_{r . i+}^{\max }$

De esta forma, para determinar una condición de seguridad del reencendido del arco, es necesario determinar el valor $U_{r . i+}^{\max }$, voltaje máximo del pico de reencendido durante el selııcııı positivo. Esto puede hacerse mediante el empleo de técnicas estadísticas. Investigaciones muestran que la distribución de los valores de $U_{r, i+}$ en la soldadura con electrodos está gobernada generalmente por una ley normal. Luego el valor $U_{r, i+}^{\max }$, puede ser determinado con una fiabilidad de aproximadamente un 99 \%, usando la Ecuación 5: 
$U_{r . i+}^{\max }=\bar{U}_{r . i+}+3 S_{U^{+}}^{r . i}$

donde $S_{U^{+}}^{r . i}$ es la desviación estándar del voltaje $U_{r . i+}$, como se muestra en la Ecuación 6.

$S_{U^{+}}^{r . i}=\sqrt{\frac{\sum_{i=1}^{n}\left(U_{r . i+}-\overline{U_{r . i+}}\right)^{2}}{(n-1)}}$

Nótese que en estos casos se asume que la distribución probabilística de los valores de voltaje de arco se puede considerar una distribución normal o de Gauss, por lo que es posible considerar el promedio de las muestras como medida localización y a partir de ahí calcular la desviación estándar.

Otros estudios han demostrado que las distribuciones obtenidas en la soldadura de corriente alterna con electrodos revestidos presentan una distribución que no se ajusta a un modelo normal, por lo que se ha propuesto el empleo de técnicas no paramétricas de procesamiento estadístico [9,10].

\section{Método C (Alejandro)}

Se emplea un índice basado en la determinación de $\mathrm{B}^{+}$, a partir del empleo de la misma definición de Farias (Ecuación 1) pero utilizando como medida de tendencia central la mediana de la distribución $\mathrm{M}\left(\mathrm{B}^{+}\right)$en lugar de la media, lo cual no depende del tipo de distribución y como medida de dispersión emplea la Median Absolute Deviation (MAD) como se detalla en la Ecuación 7.

$\mathrm{MAD}=$ mediana $($ valor absoluto $(\mathrm{X}-\operatorname{mediana}(\mathrm{X})))$

donde cada valor $\mathrm{X}$ es restado del valor de la mediana de la distribución del parámetro $\mathrm{X}$ y posteriormente se calcula la mediana de la distribución del conjunto de tales diferencias.

El objetivo del presente trabajo es comparar los resultados obtenidos durante la aplicación de tres métodos de evaluación del proceso de transferencia de carga eléctrica a través del arco de soldadura, publicados en la literatura especializada, considerando resultados obtenidos mediante análisis de la geometría del cordón de soldadura y la variabilidad de la distancia entre frentes de solidificación con ayuda del empleo de técnicas metalográficas.

\section{Materiales y Métodos}

Los electrodos rutílicos presentan un desempeño eléctrico operativo de referencia respecto al resto de los electrodos revestidos [11] por lo que se escoge para el estudio un grupo de electrodos E6013 según la norma AWS-A5.1, de fabricación española de $4 \mathrm{~mm}$ de diámetro y un rango de corriente definido por el fabricante de entre 120 a $160 \mathrm{~A}$.

Antes de someter los electrodos a prueba se realizaron procedimientos de: identificación del electrodo; inspección visual en busca de poros o grietas en el revestimiento; medición del diámetro de la varilla y el revestimiento; observación del grado de excentricidad del revestimiento empleando un pie de rey de $0,05 \mathrm{~mm}$ de precisión. También se realizó la medición de la longitud del electrodo. La determinación del peso total del electrodo, el peso de la varilla y el peso del revestimiento fueron realizadas con una balanza digital certificada.

Las muestras para la caracterización fueron tomadas de forma aleatoria en base a un muestreo sin reposición dentro de paquetes de embalaje comercial lo que garantiza que los electrodos mantengan sus composiciones y propiedades en rangos establecidos por normas, tanto en su revestimiento como en su núcleo metálico; lo cual verifica el sistema de calidad de la empresa productora durante la certificación del producto.

Todos los electrodos empleados en la experimentación fueron sometidos a un proceso de secado al horno a una temperatura de $120{ }^{\circ} \mathrm{C}$ durante una hora, para minimizar el efecto de la humedad en el revestimiento [11].

\subsection{Caracterización de materiales}

El proceso de caracterización se desarrolló mediante

Tabla 1. Composición química de los componentes del revestimiento

\begin{tabular}{|c|c|c|c|c|c|c|c|c|c|c|c|c|c|}
\hline Óxido & $\mathrm{SiO}_{2}$ & $\mathrm{Al}_{2} \mathrm{O}_{3}$ & $\mathrm{Fe}_{2} \mathrm{O}_{3}$ & $\mathrm{TiO}_{2}$ & $\mathrm{CaO}$ & $\mathrm{MgO}$ & $\mathrm{Na}_{2} \mathrm{O}$ & $\mathrm{K}_{2} \mathrm{O}$ & $\mathrm{P}_{2} \mathrm{O}_{5}$ & $\mathrm{MnO}$ & $\mathrm{SO}_{3}$ & PPI & Total \\
\hline$\%$ & 28,63 & 0,63 & 3,3 & 38,34 & 6,23 & 0,24 & 0,18 & 4,37 & 0,04 & 5,34 & 0,13 & 10,10 & 97,53 \\
\hline
\end{tabular}

Tabla 2. Composición mineralógica del revestimiento

\begin{tabular}{|c|c|c|c|c|}
\hline Mineral & Rutilo & Leucoxeno & Carbonato & Aglutinante \\
\hline$\%$ & 30 & 1 & 39 & 30 \\
\hline
\end{tabular}

Tabla 3. Composición química del metal de las probetas de ensayo

\begin{tabular}{|c|c|c|c|c|c|}
\hline Elemento & $\mathrm{C}$ & $\mathrm{Si}$ & $\mathrm{Mn}$ & $\mathrm{P}$ & $\mathrm{S}$ \\
\hline$\%$ & 0,136 & 0,01 & 0,979 & 0,05 & 0,001 \\
\hline
\end{tabular}

Tabla 4. Composición química del alma del electrodo

\begin{tabular}{|c|c|c|c|c|c|}
\hline Elemento & $\mathrm{C}$ & $\mathrm{Mn}$ & $\mathrm{Si}$ & $\mathrm{S}$ & $\mathrm{P}$ \\
\hline$\%$ & 0,09 & 0,30 & 0,10 & 0,03 & 0,03 \\
\hline
\end{tabular}


procedimientos certificados por normas ISO, en laboratorios especializados, obteniéndose los resultados de los análisis químicos y mineralógicos de la composición del revestimiento (Tablas 1 y 2).

Como metal base para las probetas, fue elegido un acero de bajo contenido de carbono del tipo CT-3 conforme normas Rusas (Tabla 3), caracterizado por el método de espectroscopia de emisión atómica. La composición química del acero empleado en el núcleo de los electrodos fue determinada coincidiendo con la composición definida para la clasificación E6013.

\subsection{Planeación experimental}

Para la organización experimental se utilizó un diseño factorial [10] simple con un factor corriente variando en tres niveles $\left(1^{3}\right)$, utilizando dos réplicas $(3 \times 3 \times 3)$, por lo que se realizaron en total 9 experimentos, en que la corriente de soldadura fue controlada mediante un ajuste variable en el transformador de soldadura AIRCO de fabricación americana y establecida cuidadosamente en valores de 125, 140 y $160 \mathrm{~A}$.

Tabla 5. Diseño factorial simple $1^{3}$ con dos réplicas

\begin{tabular}{|c|c|c|c|}
\hline Corriente (A) & \multicolumn{3}{|c|}{ Identificación del experimento } \\
\hline 125 & E1 & E2 & E3 \\
\hline 140 & E4 & E5 & E6 \\
\hline 160 & E7 & E8 & E9 \\
\hline
\end{tabular}

\subsection{Procedimiento experimental}

El experimento consistió en realizar el depósito de un cordón de soldadura de un electrodo en cada uno de los nueve experimentos, en probetas en posición plana, utilizando un dispositivo de alimentación por gravedad, ajustando, según especificaciones del fabricante, la corriente en los valores de 125, 140 y 160 A según el caso. Durante el tiempo en que transcurre cada experimento se registran digitalmente los voltajes y corrientes de soldadura, por medio de un acondicionador de señales acoplado a un osciloscopio digital profesional con un ancho de banda de $100 \mathrm{MHz}$ y una capacidad máxima de muestreo de 2,5 Gigamuestras por segundo (GS/s). El muestreo de las señales de voltaje y corriente de soldadura se produjo a razón de 5000 muestras por segundo. Para evitar la influencia del operador se alimenta el electrodo con el empleo de un dispositivo de alimentación por gravedad [1].

\subsection{Conductividad durante los picos de reencendido}

En el tiempo en que transcurrieron los experimentos se obtuvieron cientos de miles de muestras de las cuáles se seleccionaron las primeras 84000 en cada experimento. Las señales de voltaje y corriente sincronizadas en tiempo (Figura 1) fueron analizadas con el paquete de herramientas Procesamiento Digital de Señales (PDS) de Matlab 10.0.

La conductividad durante el reencendido del arco se calcula de manera automatizada a partir de la determinación del voltaje y la corriente promedios durante el reencendido y su tiempo de duración ((1), Figura 1), mediante un programa desarrollado con este fin: Una vez detectado el borde de subida de la señal de voltaje, (es decir, una vez que la misma pasa de un valor negativo a un valor positivo de voltaje), se toma como referencia el primer valor positivo, después de la ocurrencia del último valor negativo, guardando el instante de tiempo en que esto ocurre. Luego debe irse incrementando el puntero de la señal de voltaje tomando como referencia el valor que le sigue, mientras el valor siguiente supere el valor de referencia o presente; lo que evidencia una subida de la señal y esta condición se mantendrá hasta tanto el valor siguiente sea menor que el de referencia. En este punto, el valor de referencia debe ser el máximo valor de voltaje del semiciclo, por lo que se compara con el valor de la Raíz Cuadrática Media del voltaje, de sus siglas en inglés (Root Mean Squared), discriminando falsos picos de reencendido.

Para la detección del reinicio del arco se repite el proceso

Tabla 6. Resultados de la aplicación de los métodos A, B y C a los nueve conjuntos de datos resultados de la experimentación

\begin{tabular}{|c|c|c|c|c|c|c|c|c|c|c|}
\hline \multirow{2}{*}{\multicolumn{2}{|c|}{$\begin{array}{l}\text { Método } \\
\text { Muestra }\end{array}$}} & \multicolumn{3}{|c|}{ A } & \multicolumn{3}{|c|}{ B } & \multicolumn{3}{|c|}{ C } \\
\hline & & $\overline{B^{+}}$ & $\sigma_{B+}$ & $R B^{+}$ & $\bar{U}_{r, i+}$ & $3 S_{U^{+}}^{r . i}$ & $U_{r . i+}^{\max }$ & $M\left(B^{+}\right)$ & $M A D\left(B^{+}\right)$ & $C P$ \\
\hline \multirow{3}{*}{$125 \mathrm{~A}$} & $\mathrm{E}_{1}$ & 1658,5 & 1752,0 & 0,95 & 36,27 & 22,45 & 58,72 & 1152,9 & 422,91 & 311 \\
\hline & $\mathrm{E}_{2}$ & 2188,1 & 3728,7 & 0,58 & 36,95 & 22,91 & 59,86 & 1169,1 & 505,70 & 307 \\
\hline & $\mathrm{E}_{3}$ & 1995,4 & 2695,8 & 0,74 & 36,46 & 22,64 & 59,10 & 1159,2 & 455,50 & 297 \\
\hline \multirow{2}{*}{$140 \mathrm{~A}$} & $\mathrm{E}_{4}$ & 2036,5 & 3374,8 & 0,60 & 35,02 & 18,74 & 53,76 & 1291,8 & 387,93 & 261 \\
\hline & $\mathrm{E}_{5}$ & 2001,2 & 2355,7 & 0,85 & 35,28 & 17,88 & 53,16 & 1311,4 & 388,44 & 287 \\
\hline \multirow{4}{*}{$160 \mathrm{~A}$} & $\mathrm{E}_{6}$ & 2063,8 & 2451,9 & 0,84 & 34,38 & 21,53 & 55,91 & 1345,6 & 446,61 & 231 \\
\hline & $\mathrm{E}_{7}$ & 4694,7 & 7985,8 & 0,59 & 34,48 & 18,57 & 53,05 & 2081,30 & 1340,78 & 125 \\
\hline & $\mathrm{E}_{8}$ & 3353,2 & 4005,2 & 0,84 & 34,59 & 16,98 & 51,57 & 2110,48 & 1241,36 & 110 \\
\hline & $\mathrm{E}_{9}$ & 2589,4 & 3241,4 & 0,78 & 34,10 & 14,80 & 48,9 & 2005,0 & 1063,77 & 116 \\
\hline
\end{tabular}


descrito anteriormente pero ahora la condición para el avance considera que el valor de referencia sea mayor que el que le sigue. La condición que detiene el algoritmo es que el valor de referencia sea menor que el que le sigue y en este punto se asume que comienza el período de arco, por lo que se resta el valor de tiempo del valor guardado anteriormente para definir el tiempo de reencendido del arco. El voltaje pico debe ser mayor que el voltaje promedio del semiciclo, para ser considerado válido. El algoritmo, como resultado, genera un fichero que contiene los valores de voltaje pico y los valores de conductividad por unidad de tiempo.

\section{Resultados obtenidos}

\subsection{Comparación de métodos}

Las datas experimentales obtenidas han sido procesadas por un software capaz de detectar los picos de reencendido positivos, calculando la conductividad promedio $\mathrm{B}^{+}$de cada pico, constituyendo un conjunto de datos característico de cada uno de los 9 experimentos. Cada uno de estos conjuntos es entonces procesado estadísticamente según los algoritmos definidos A, B y C con ayuda de Matlab 10.0. Los resultados del procesamiento permiten estudiar el comportamiento de los métodos evaluados (Tabla 6).

En la Tabla $6, \overline{B^{+}}$es la media del parámetro $B^{+}, \sigma_{B+}$ es la desviación estándar de $B^{+} ; M\left(B^{+}\right)$es la mediana de $B^{+}$ y $M A D\left(B^{+}\right)$es la Desviación Absoluta de la Mediana de $B^{+}$. Las tres variables se dan en S/s (Siemen/segundo). Por su parte $\bar{U}_{r . i+}$ es el voltaje promedio de reencendido positivo del arco $3 S_{U^{+}}^{r . i}$;es tres veces la desviación estándar del voltaje de reencendido positivo y $U_{r, i+}^{\max }$ es el voltaje máximo del pico de reencendido. Estos tres índices se dan en volt (V). Por otra parte $\mathrm{RB}^{+}$es la regularidad de la Conductividad (Ecuación 2) mientras

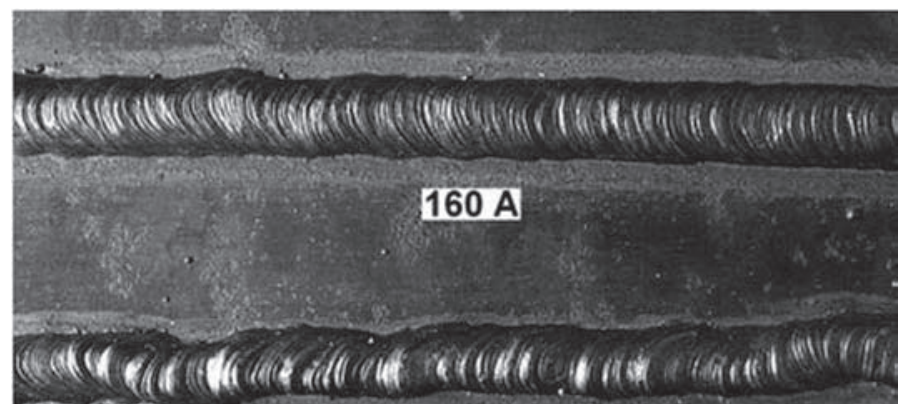

$140 \mathrm{~A}$

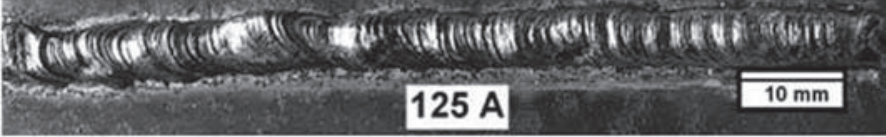

Figura 2. Fotografía de una de las probetas experimentales con tres cordones depositados a 125, 140 y $160 \mathrm{~A}$ que $\mathrm{CP}$ es la cantidad de picos de reencendido. Los índices y $\mathrm{CP}$ son adimensionales.

\subsection{Morfología de los cordones}

En las condiciones experimentales dadas la velocidad de soldadura varía en función de la intensidad de la corriente que determina la velocidad de fusión del electrodo puesto que el ángulo de incidencia del electrodo sobre la placa a soldar, definido con el dispositivo de alimentación por gravedad, es el mismo en todos los experimentos y los electrodos evaluados son prácticamente idénticos. Tres de los cordones fueron depositados en cada una de las tres probetas de 300 x150 mm (Figura 2) en diferentes corrientes (160,140 y $125 \mathrm{~A})$.

Luego de la realización de varios cortes transversales a la probeta, pulidos y el atacados con NITAL (2\%) se midieron, empleando el microscopio y con el auxilio de técnicas óptico digitales (Figura 3), las características morfológicas de los cordones determinando un grupo de parámetros: Ancho (An), Altura de refuerzo (Ar), Distancia entre conchas (Dc) Penetración (Pe) y Zona Afectada por el calor (ZAC); calculando la desviación estándar de cada parámetro medido (Dv) (Tabla 7).

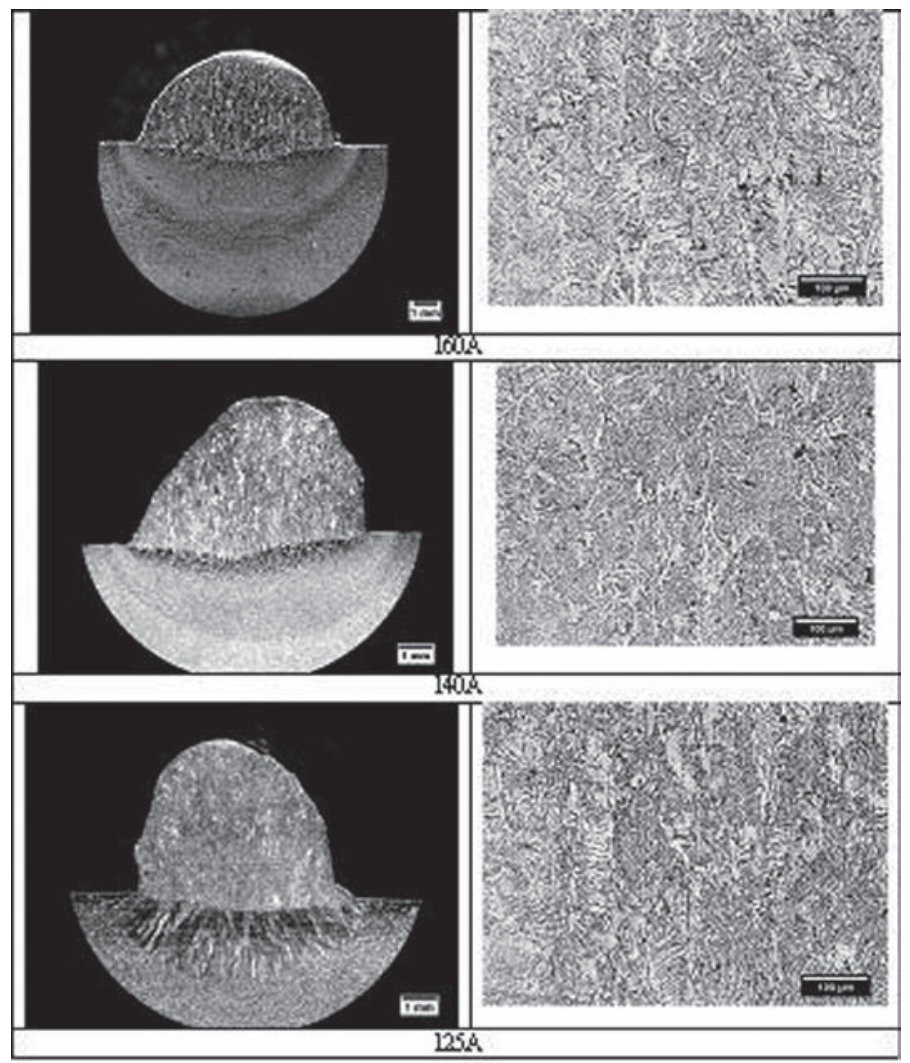

Figura 3. Macrografía de la unión soldada (izquierda) y micrografía en la zona de fusión (derecha); para cordones obtenidos en los regímenes de 125, 140 y 160 A 
Tabla 7. Valores promedios y desviación estándar de los parámetros morfológicos del cordón

\begin{tabular}{|c|c|c|c|c|c|c|c|c|c|}
\hline I(A) & An & Dv & Dc & Dv & Ar & Dv & Pn & Dv & ZAC \\
\hline 125 & 6,11 & 0,398 & 0,057 & 0,018 & 4,34 & 0,251 & 0,43 & 0,092 & 1,42 \\
\hline 140 & 7,30 & 0,280 & 0,052 & 0,014 & 4,17 & 0,146 & 0,52 & 0,059 & 1,92 \\
\hline 160 & 8,17 & 0,145 & 0,046 & 0,012 & 3,83 & 0,101 & 0,70 & 0,041 & 2,65 \\
\hline
\end{tabular}

Obs: (An) es el Ancho del cordón, (Ar) es la Altura de refuerzo, (Dc) es la Distancia entre conchas, (Pe) es la Penetración, (ZAC) es la Zona Afectada por el calor y (Dv) es la desviación estándar (todos expresados en milímetros, según el Sistema Internacional de medidas)

\subsection{Análisis de resultados}

La estimación de las medidas de tendencia central y dispersión en una distribución probabilística empírica mediante el empleo de técnicas no paramétricas, exige que la misma posea un alto grado de similitud con alguna de las distribuciones probabilísticas conocidas (modeladas matemáticamente). El empleo del promedio o la media de una distribución como medida de tendencia central es solamente correcto cuando se prueba que la misma posee una distribución de Gauss o Normal, al igual que el empleo de la desviación estándar como medida de dispersión de la distribución $[9,10]$.

El método de Farías (A) asume que las distribuciones probabilísticas de conductividad durante el pico de reencendido se pueden tratar como distribuciones normales, sin embargo, la aplicación de pruebas de normalidad a las distribuciones empíricas de este parámetro demuestra exactamente lo contrario (Tabla 8).

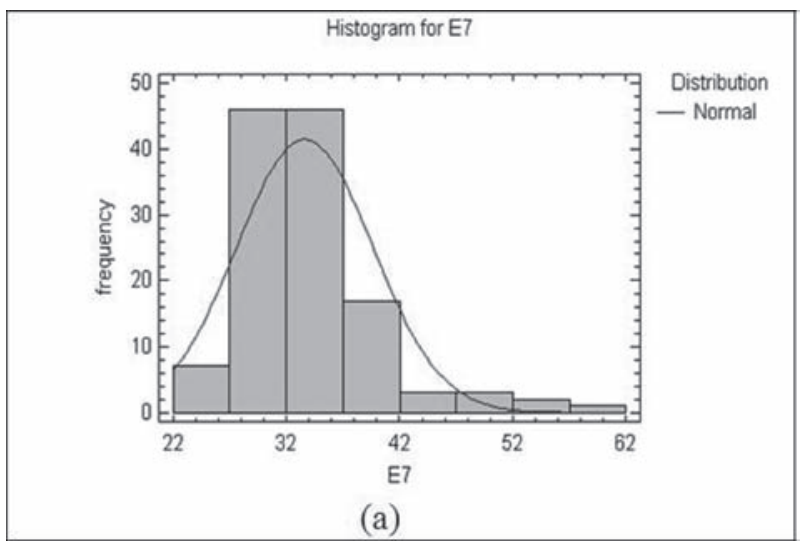

Tabla 8. Resultado de la aplicación de pruebas de normalidad a las 9 distribuciones empíricas de $\mathrm{B}^{+}$

\begin{tabular}{|c|c|c|}
\hline \multicolumn{3}{|c|}{ Prueba de normalidad (Shapiro-Wilk W ) } \\
\hline Experimento & Estadística & Valor de probabilidad \\
\hline $\mathrm{E}_{1}$ & 0,545343 & 0,0 \\
\hline $\mathrm{E}_{2}$ & 0,498047 & 0,0 \\
\hline $\mathrm{E}_{3}$ & 0,501287 & 0,0 \\
\hline $\mathrm{E}_{4}$ & 0,328759 & 0,0 \\
\hline $\mathrm{E}_{5}$ & 0,481415 & 0,0 \\
\hline $\mathrm{E}_{6}$ & 0,482542 & 0,0 \\
\hline $\mathrm{E}_{7}$ & 0,465522 & 0,0 \\
\hline $\mathrm{E}_{8}$ & 0,594531 & 0,0 \\
\hline $\mathrm{E}_{9}$ & 0,476351 & 0,0 \\
\hline
\end{tabular}

La prueba de Shapiro-Wilk W se basa en la comparación de las características de los cuartiles de la distribución normal con los cuartiles de la data empírica [12]. Como el valor de probabilidad más pequeño obtenido de la ejecución de la prueba es menor que 0,05 , podemos descartar la idea de que las datas sometidas a prueba provienen de una distribución normal con grado de certidumbre del $95 \%$.

Por su parte las distribuciones probabilísticas empíricas del pico de voltaje de reencedido en cualquiera de los nueve experimentos fue sometida a pruebas de normalidad (ShapiroWilk W) demostrándose con un $95 \%$ de certidumbre que las datas obtenidas no pueden ser adecuadamente modeladas por una distribución normal (Figura 4).

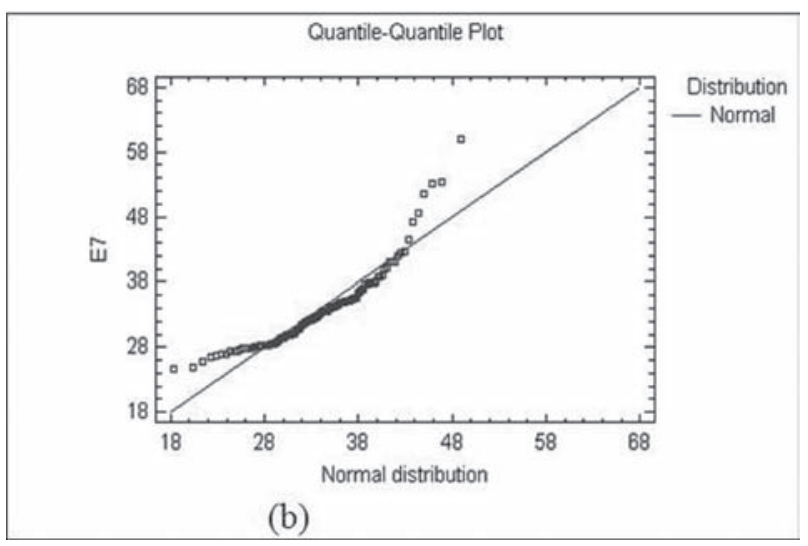

Figura 4. (a) Histograma de Voltaje pico de reencendido $\left(U_{r . i+}\right)$ en el experimento E7 (Tabla 5); (b) Comparación de cuartiles de la distribución empírica con la normal (la magnitud del eje horizontal se encuentra en volt (V)).

Tabla 9. Valores promedios de la (Tabla 6)

\begin{tabular}{|c|c|c|c|c|c|c|c|c|c|}
\hline Métodos & \multicolumn{3}{|c|}{$\mathrm{A}$} & \multicolumn{3}{|c|}{$\mathrm{B}$} & \multicolumn{3}{c|}{$\mathrm{C}$} \\
\hline Muestra & $\overline{B^{+}}$ & $\sigma_{B+}$ & $R B^{+}$ & $\bar{U}_{r . i+}$ & $3 S_{U^{+}}^{r . i}$ & $U_{r . i+}^{\max }$ & \multirow{2}{*}{$M\left(B^{+}\right)$} & $M_{A D\left(B^{+}\right)}$ & $C P$ \\
\hline $125 \mathrm{~A}$ & 1947,33 & 2725,5 & 0,76 & 36,56 & 22,67 & 59,23 & 1160,4 & 461,37 & 305 \\
\hline $140 \mathrm{~A}$ & 2033,83 & 3820,33 & 0,76 & 34,9 & 19,38 & 54,28 & 1316,27 & 407,66 & 259,66 \\
\hline $160 \mathrm{~A}$ & 3545,77 & 5077,47 & 0,74 & 34,39 & 16,78 & 51,17 & 2065,59 & 1215,3 & 117 \\
\hline
\end{tabular}


Este resultado indica que los resultados obtenidos por medio de métodos que asuman normalidad en estas distribuciones empíricas no pueden fundamentarse desde el punto de vista de un riguroso examen estadístico; sin embargo como en el método $\mathrm{C}$ se emplea un método no paramétrico que es independiente del tipo de distribución, desde el punto de vista estadístico son mucho más significativos los valores obtenidos. Como es posible observar (Tabla 9), la regularidad en el caso del método A, $R B^{+}$no permite diferenciar la estabilidad en los diferentes regímenes de trabajo sin embargo el índice $M A D\left(B^{+}\right)$es menor para el caso de $140 \mathrm{~A}$. Los índices del método B son muy consistentes, aunque las distribuciones hayan sino diferentes de la distribución normal y sugieren, de acuerdo con los resultados del estudio metalográfico que el régimen más estable es el de $160 \mathrm{~A}$.

Aunque las distribuciones de los voltajes pico de reencendido no sean normales se observa una disminución del voltaje $U_{r . i+}^{\max }$ medida que se aumenta el nivel de corriente proporcionalmente a la temperatura del arco, mientras mayor es el régimen menor voltaje pico de reencendido. Este resultado resulta acorde con los principios físicos del arco eléctrico de soldadura.

Por otra parte, este resultado concuerda con el hecho de que la mediana de la conductividad promedio durante el reencendido del arco $\mathrm{M}(\mathrm{B}+)$ sea mayor en el caso de $160 \mathrm{~A}$, puesto que $\mathrm{B}+$ es inversamente proporcional al tiempo de reencendido y la relación corriente sobre tensión es mayor, lo que indica una mayor facilidad de encendido. Este resultado es coherente con el hecho de que el voltaje pico de reencendido haya sido menor (Tabla 9) indicando que se requiere menor energía para el reinicio del arco, puesto que el voltaje y el tiempo son inversamente proporcionales a $B^{+}$, ambos constituyen factores importantes a tener en cuenta (Ecuación 1).

Paradójicamente la máxima dispersión $M A D\left(B^{+}\right)$ocurre en el régimen de $160 \mathrm{~A}$, indicando estadísticamente que el régimen de mayor dispersión en la conductividad coincide con el que más fácilmente reenciende el arco (mayor conductividad). Este resultado se debe posiblemente a que el proceso de reencendido estuviera ocurriendo más rápida y fácilmente producto de la mayor severidad del régimen afectando la dispersión estadística de este parámetro que en estas condiciones no debe considerarse un índice confiable para valorar el proceso de transferencia de carga. El resultado que expresa que la cantidad de picos de reencendido $(C P)$ sea mucho menor en este régimen (Tabla 9) y la conductividad $M\left(B^{+}\right)$sea mayor viene a concluir que efectivamente hay más estabilidad general en el transporte de carga eléctrica en este régimen, junto con el índice de voltaje de reencendido máximo empleado en el método $\mathrm{B}$, aunque estadísticamente este resultado pueda ser cuestionado. Sería interesante comparar este resultado con la MAD del voltaje pico de reencendido.

El estudio metalográfico arroja (Tabla 7) que en el régimen de $125 \mathrm{~A}$ los cordones presentan menores valores en parámetros como An, Pn y ZAC mientras Ar y Dc son mayores. Las desviaciones estándares de estos parámetros son mayores en este régimen, indicando que hay mayor inestabilidad del proceso comparado con los regímenes de 140 y 160 A. Para los cordones del régimen de 160 A se aprecian los mayores valores de An, Pn y ZAC, mientras Ar y Dc son menores. Las desviaciones estándares son también menores indicando que el proceso ha ocurrido más establemente en la práctica experimental de acuerdo con resultados de investigaciones anteriores [4]. La microestructura obtenida en los cordones (Figura 3) es característica de depósitos realizados con electrodo revestido en aceros de bajo contenido de carbono [13]. Se aprecian microestructuras típicas tales como: Ferrita Poligonal, Ferrita Widmanstätten, Ferrita de Contorno de grano y Ferrita Alineada fundamentalmente.

Partiendo de este resultado es posible concluir que el régimen que, según los resultados del estudio del proceso de transferencia de carga eléctrica, ha sido reconocido como el más estable (160 A) también ha sido reconocido como el de mayor estabilidad en mediante el análisis metalográfico, lo cuál ha sido corroborado además por criterios de expertos, inspección visual y observación durante la ejecución del proceso de experimentación.

\section{Conclusiones}

1 - Las pruebas de normalidad realizadas demostraron que las distribuciones probabilísticas empíricas de la conductividad durante el reencendido $\left(\mathrm{B}^{+}\right)$y las voltajes del pico de reencendido (Ur.i+) no presentaron una distribución normal. Por lo que los resultados obtenidos producto de la aplicación de los métodos A y B no son confiables desde el punto de vista estadístico, aunque pudieran tener alguna importante significación de interés cualitativo.

2 - Debido a las características de las distribuciones probabilísticas de los parámetros evaluados para la obtención de resultados cuantitativos adecuados desde el punto de vista ingenieril es más factible el empleo del procesamiento no paramétrico propuesto en el Método C. De los parámetros evaluados para valorar el proceso de transferencia de carga eléctrica la mediana de la conductividad de los picos de reencendido $(\mathrm{M}(\mathrm{B}+))$ y la cantidad de picos de reencendido (CP) (Tabla 9) se presentan como los índices más representativos tanto desde el punto estadístico como fenomenológico.

3 - Los resultados del análisis metalográfico (Tabla 7) y del análisis de estabilidad del proceso de transferencia de carga eléctrica (Tabla 9) coinciden en que el régimen de $160 \mathrm{~A}$ es el más estable, corroborándose la validez del método empleado.

\section{Agradecimientos}

Quisiera agradecer sinceramente a todos los que de una forma u otra han colaborado para la realización de este trabajo y a otros sin los cuales el mismo no hubiera tal vez podido culminarse satisfactoriamente o ni siquiera comenzarse. Especialmente destaco la colaboración desinteresada de los técnicos Jorge V Miguel Oria y Alexis Delgado Gómez en las tareas de procesamiento metalográfico de las muestras y la asistencia a la instalación experimental respectivamente; por el alto nivel de profesionalidad y seriedad demostrado durante el transcurso de la presente investigación. A los compañeros Drs. Rafael Quintana Puchol y Carlos René Gómez Pérez por sus consejos 
en los momentos iniciales del trabajo. A los investigadores que me precedieron en este tema con tan valiosos aportes: Drs. Vladimir Ponomarev y JP Farias, al consejo editorial de la más importante revista Latinoamericana especializada en soldadura (Soldagem e Inspeção) y en general a toda la comunidad científica relacionada el campo de la soldadura en el ámbito cubano y mundial.

\section{Referencias Bibliográficas}

[1] GARCIA-RODRIGUEZ, A. Evaluación de la estabilidad eléctrica específica de electrodos revestidos. [Tesis Doctoral]. ISBN 978-959-250-679-4, Feijoo. Universidad Central "Marta Abreu” de Las Villas. Santa Clara, Villa Clara, CUBA. 2011.

[2] LANCASTER, J.F. The physics of welding. 2a ed. Oxford: International Institute of Welding; 1986.

[3] GAPONOV, V.I. Física electrónica: MIR; 1968.

[4] GARCIA RODRIGUEZ, A, GOMEZ PEREZ, C.R.. Criterios sobre algunas Metodologías de Caracterización Operativa de Electrodos Revestidos en la Soldadura en Modo de Transferencia Metálica por Cortocircuitos. Soldagem \& Inspeçao. 2008; 13(2): 141.

[5] FARIAS, J.P. Magnésio Metálico como Componente do Revestimento na Soldagem com Eletrodos ao C-Mn-Ni, [Tesis doctoral]. Universidad Federal de Santa Catarina, Brazil; 1993. [6] FROLOV, V. Teoría de los procesos de soldadura. Moscú: E. Superior; 1989.

[7] PONOMAREV, V. Arc welding process statistical analysis. Methodical Approaches, Analysis Conceptions, Experiences; JOM-Institute, DTU-Helsingor, Denmark, 158 p, 1997.

[8] SOUZA, M.S. Avaliação de um Sistema Computadorizado para Controle de Qualidade de Eletrodos Revestidos. [Tesis de maestría], Universidad Federal de Uberlandia, Brazil; 1995.

[9] SACHS, L. Applied statistics: A Handbook of Techniques: Springer-Verlag; 1984.

[10] OSTLE, B. Estadística Aplicada. Ministerio de Cultura: Editorial Científico-Técnica; 1979.

[11] ANSI/AWS.ANSI/AWS 5.1-91. Specification for carbon steel electrodes for shielded metal arc welding, Standard 5.191, Miami, Fl: American National Standards Institute/American Welding Society; 1991.

[12] ROYSTON, J.P. A toolkit for testing for non-normality in complete and censored samples. The statistician. 1993; 42: 3742.

[13] LAMET, et al. ASM Hanbook.Welding Brazing and Soldering. ASM International. 1993. 